\title{
Spatial patterning of gene expression using surface-immobilized recombinant adenovirus
}

\author{
Dana M. Pirone • Lixin Qi • Henry Colecraft • \\ Christopher S. Chen
}

(C) Springer Science + Business Media, LLC 2008

\begin{abstract}
Spatially patterned gene expression drives tissue organization and is a critical determinant of tissue function. Approaches in functional tissue engineering will require not only the spatial organization of cells but also control of their gene expression patterns. We report a method to generate patterns of gene expression within a monolayer of cells by using surface-immobilized recombinant adenovirus. This study represents a new approach to engineering tissues that relies on controlling spatial patterns of gene expression, and can be used independently or in combination with positioning of different cell types. This technique may have broad applications in biotechnology including tissue engineering and targeted gene delivery.
\end{abstract}

Keywords Adenovirus · Alkanethiols · SAMs · Patterning · Gene expression

\section{Introduction}

A central goal in cell and tissue engineering is to recapitulate tissue structures such that the organization of the engineered tissue reflects that of native tissue. Because the spatial organization of cells defines the proximity of individual cells to various microenvironmental cues, spatial

D. M. Pirone $\cdot$ C. S. Chen $(\bowtie)$

Department of Bioengineering, University of Pennsylvania,

210 S. 33rd Street, Skirkanich 510,

Philadelphia, USA

e-mail: chrischen@seas.upenn.edu

L. Qi $\cdot$ H. Colecraft

Department of Biomedical Engineering,

Johns Hopkins University School of Medicine,

720 Rutland Avenue,

Baltimore, MD 21205, USA structure is thought to be a key element in defining appropriate spatial patterns of cell behavior within the tissue. This patterning of cell types and functions within a tissue is the ultimate aim of tissue engineering efforts. As such, the engineered tissue would not only look like its endogenous counterpart, but also be functionally indistinct.

Toward this end, there have been numerous approaches developed to control the placement and organization of single cells as well as groups of cells (Albrecht et al. 2006; Chen et al. 1997; Nelson et al. 2005). In some cases, this has led to the regulation of certain cell functions, such as cell proliferation (Pirone et al. 2006) or differentiation (McBeath et al. 2004; Singhvi et al. 1994). In one example, hepatocytes rapidly lose differentiated function when cultured in vitro, but if they are geometrically patterned to make contact with fibroblasts, the hepatocytes are induced to maintain differentiated functions such as production of albumin and expression of metabolic enzymes (Bhatia et al. 1998). In essence, geometric patterning of single or multiple cells provides one, albeit indirect, means to regulate the patterns of gene expression involved in producing desired cell functions.

Directly modulating spatial patterns of gene expression within a cell population may provide an alternative, more direct means to pattern cell function. In vivo, patterning the expression of key master regulator genes within tissues is a principal developmental mechanism that gives rise to patterned differentiation of cell types and behaviors (Serls et al. 2005). By controlling which and how much mRNA and protein is produced, one can regulate many basic functions, including proliferation, migration, and differentiation. Localized regions of such gene expression guide critical steps of tissue formation including anterior/posterior organization, left-right symmetry or asymmetry, and limb bud site selection (reviewed in (Kawakami et al. 2006; 
Maeda et al. 2007; Olson 2006)). Thus, changing patterns of gene expression is a principal control point of cell function that forms tissue boundaries and specifies the unique structures that become organs and tissues (Abzhanov and Tabin 2004; Hu et al. 2003). This leads to the notion that patterning gene expression, rather than patterning the cells themselves, may be a critical, yet underexplored, point of control for tissue engineering.

Early studies demonstrated that gene expression could be patterned in culture via a reverse transfection procedure wherein plasmid DNA is immobilized on a substrate onto which a monolayer of cells are subsequently grown (Ziauddin and Sabatini 2001). The application of this approach to tissue engineering has been limited because plasmid transfection is effective in only a few cell types - a list that does not include primary cells, which are key players for tissue engineering. To circumvent the limitations of plasmid-based transfection, biologists have developed numerous viral vectors that have substantially higher efficiency in targeting cells (Imperiale and Kochanek 2004). Lentiviruses in particular have been immobilized onto a glass surface using a conventional microarrayer, but the surface chemistry underlying such immobilizations has not been well explored (Bailey et al. 2006).

Here, we sought to investigate whether we could pattern gene expression within a monolayer of cells by using surface-immobilized recombinant adenovirus to direct gene expression to specific regions. We show that specific surface chemistries can enhance immobilization of infectious viral particles, and then use this approach to demonstrate patterned gene expression. This study represents a new approach to engineering tissues that relies on controlling spatial patterns of gene expression, rather than controlling spatial positioning of different cell types.

\section{Materials and methods}

Cell culture Bovine Pulmonary Artery Endothelial Cells (VEC Technologies) were cultured in low glucose Dulbecco's modified Eagle's medium (DMEM) containing $2 \mathrm{mM}$ glutamine, 100 units $/ \mathrm{mL}$ penicillin, $100 \mathrm{mg} / \mathrm{mL}$ streptomycin and $5 \%$ bovine serum (all from Invitrogen). Endothelial cells were maintained in a humidified $10 \% \mathrm{CO}_{2}$ incubator. Adult human Mesenchymal Stem Cells (hMSC) were obtained from Lonza, Inc. and grown in low glucose DMEM supplemented with $10 \%$ fetal bovine serum, $2 \mathrm{mM}$ glutamine, 100 units $/ \mathrm{mL}$ penicillin, $100 \mathrm{mg} / \mathrm{mL}$ streptomycin (all from Invitrogen). hMSCs were maintained in a humidified $5 \% \mathrm{CO}_{2}$ incubator.

Generation of recombinant adenovirus GFP recombinant adenovirus was constructed using the AdEasy XL system (Stratagene) according to manufacturer's instructions.
Briefly, the pShuttle-IRES-GFP1 vector was transformed into BJ5183-AD-1 bacterial cells. Following homologous recombination, the resulting plasmid was linearized and used to transfect HEK293 cells. Recombinant adenoviruses generated in the HEK293 cells were purified by $\mathrm{CsCl}_{2}$ density gradient centrifugation. Viral titers (infectious particles $/ \mathrm{mL}$ ) were determined by limiting dilution assays.

Surface chemistry modifications For the generation of SAMs, dodecanethiol (98\%), 11-mercapto-1-undecanol (97\%), and 11-mercaptoundecanoic acid (95\%) were obtained from Sigma-Aldrich. We refer to these compounds as $\mathrm{CH}_{3}$-terminated alkanethiol, $\mathrm{OH}$-terminated alkanethiol, and $\mathrm{COOH}$-terminated alkanethiol, respectively. Glass coverslips were coated with $3 \mathrm{~nm}$ of Ti to promote adhesion followed by $50 \mathrm{~nm}$ of $\mathrm{Au}$ by electron-beam evaporation. Immersion of the gold-coated coverslips in an ethanolic solution $2 \mathrm{mM}$ alkanethiol for $1 \mathrm{hr}$ functionalized the gold surface. The substrates were removed from the alkanethiol solution, washed with ethanol, then immersed in PBS.

Fabrication of elastomeric membranes Membranes were fabricated according to the method of Ostuni et al 2000 (Ostuni et al. 2000). Briefly, a template containing raised features was generated through standard photolithography. A prepolymer of poly(dimethylsiloxane) (PDMS; Sylgard 184, Dow-Corning) was spin coated onto the master and cured for $2 \mathrm{~h}$ at $60^{\circ} \mathrm{C}$. After curing, an aluminum ring was "painted" with PDMS and placed over the region containing features to allow for a rigid boundary to the membrane. After synthesis, the membranes were briefly washed with $70 \%$ ethanol in order to sterilize them for cell culture use. The membranes were sealed against a Petri dish, flooded with sterile $1 \times$ PBS and briefly exposed to vacuum to remove air blockage at the membrane-liquid interface. GFP-expressing recombinant adenovirus was then incubated with the membrane for $1 \mathrm{~h}$ at RT and washed 4 times with $1 \times$ PBS. The membrane was then removed, the Petri dish was treated with $25 \mu \mathrm{g} / \mathrm{mL} \mathrm{FN}$ and cells were seeded onto the surface.

Bead delivery Polystyrene beads $(6.0-\mu \mathrm{m}$ microspheres, Polysciences Inc.) were incubated for $1 \mathrm{~h}$ at RT with recombinant adenovirus. The beads were washed $4 \times$ with sterile $1 \times$ PBS and resuspended in cell culture medium.

\section{Results}

3.1 Surface immobilization of recombinant adenovirus

We first explored the possibility that a recombinant adenovirus (serotype 5) could be adsorbed onto the surface 
of a tissue culture dish by spotting the virus onto the surface with a pipette tip. We deposited $5 \mu \mathrm{L}$ of GFPexpressing recombinant adenovirus onto the surface of a tissue culture dish, incubated at RT for $30 \mathrm{~min}$ and washed the dish with PBS. Following washing, we plated a monolayer of human mesenchymal stem cells onto the dish. To determine whether (1) the virus immobilized to the discrete deposited region, and (2) whether the adsorbed virus was still biologically active, we observed the cells at $24 \mathrm{~h}$ for GFP expression. We observed that the cells overlaying the original spot of adenovirus expressed GFP, while the surrounding cells were not affected (Fig. 1). In

(a)
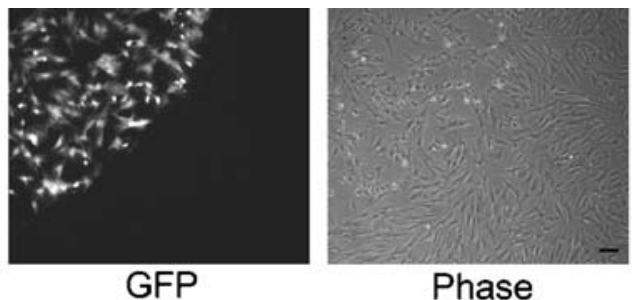

Phase

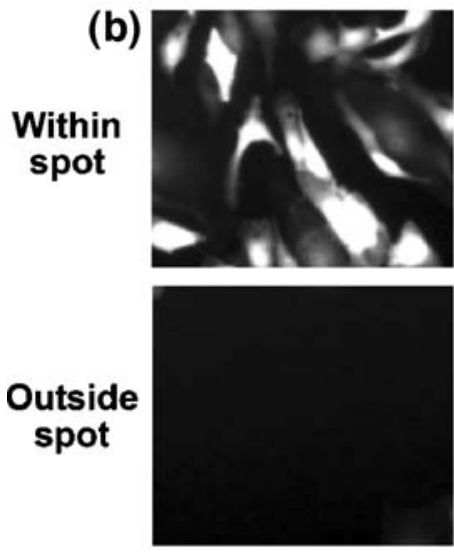

(c)

GFP

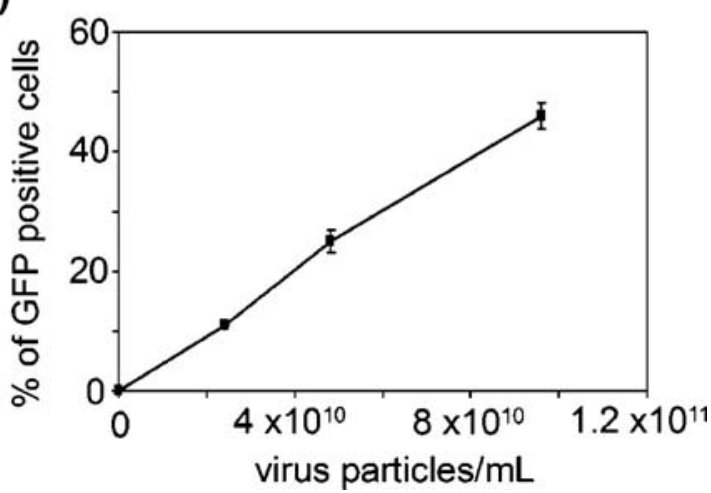

Fig. 1 Recombinant adenovirus can be immobilized onto hydrophilic surfaces to transduce human mesenchymal stem cells or bovine pulmonary artery endothelial cells (BPAEC). (a) Fluorescent and phase contrast images of MSCs plated on a tissue culture dish on which recombinant GFP adenovirus is spotted. Bar $200 \mu \mathrm{m}$. (b) Fluorescence and phase contrast images of BPAEC from within a spot of immobilized adenovirus and outside of a spot of immobilized adenovirus. Bar $50 \mu \mathrm{m}$. (c) Dose response curve of adenovirus transduction in BPAECs fact, the line of demarcation is sharp, wherein a clear edge of deposited adenovirus can be visualized by monitoring GFP-expressing cells (Fig. 1(a)). We more closely examined the penetrance of this effect at higher magnification, and observed that nearly all of the cells within the area of the adenoviral deposition expressed GFP, while none of the cells in the area outside of the viral spot expressed GFP (Fig. 1(b)). To examine whether this effect could be controlled in a dose-dependent fashion, we spotted drops of viral solution that contained different titers of recombinant adenovirus. Indeed, this effect was dose-responsive, where using a $1 \times 10^{11}$ viral particles $/ \mathrm{mL}$ solution of adenovirus resulted in around 50\% of cells expressing GFP, while using lower titers of recombinant adenovirus $\left(2.5 \times 10^{10}\right.$ viral particle/mL) produced only approximately $10 \%$ virally transduced cells (Fig. 1(c)). Together, these data suggest that recombinant adenovirus can be effectively immobilized on a tissue culture surface in a spatially controlled way. This surface-immobilized virus retained its biological function, resulting in GFP-expression only in exposed cells.

\subsection{Surface chemistry affects adenoviral immobilization}

We have demonstrated that recombinant adenovirus can be effectively immobilized on tissue culture polystyrene. To examine whether changing the surface chemistry could alter the ability of adenovirus to be immobilized and remain infectious on a surface, we used self-assembled monolayers (SAMs) of alkanethiols $\left(\mathrm{HS}\left(\mathrm{CH}_{2}\right)_{n} X\right)$ on gold as a model surface where the surface chemistry is uniform and welldefined. In these compounds the sulfur atom coordinates with a gold-coated surface, resulting in a thin semicrystalline film, whose exposed tail can have different functionalities. SAMs of alkanethiols have been routinely used to control the adsorption of proteins (Lopez et al. 1993; Mrksich et al. 1997; Prime and Whitesides 1991), thus, we tested their ability to immobilize recombinant adenovirus. As control substrates, we used two different forms of polystyrene: native, untreated polystyrene, commonly referred to as bacteriological Petri plastic, and plasma-treated polystyrene that is more hydrophilic and often used in tissue culture. As previously observed, the recombinant GFP adenovirus was readily immobilized and active on the hydrophilic plasma-treated polystyrene (tissue culture dish) surface, as cells grown on this surface expressed GFP (Fig. 2(a) and (b)). In contrast, the recombinant adenovirus did not lead to GFP expression on the more hydrophobic native polystyrene (Petri dish) (Fig. 2(a) and (b)). We then examined three different alkanethiols: one that was functionalized with carboxylic acid $(\mathrm{COOH})$, one that was functionalized with a hydroxyl $(\mathrm{OH})$ moiety, and one that was $\mathrm{CH}_{3}$-terminated for their ability to immobilize recombi- 
(a)

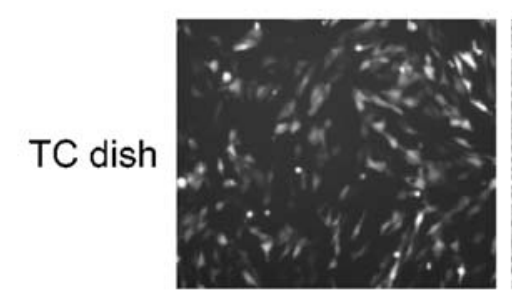

GFP

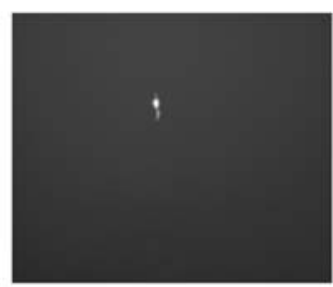

Petri dish

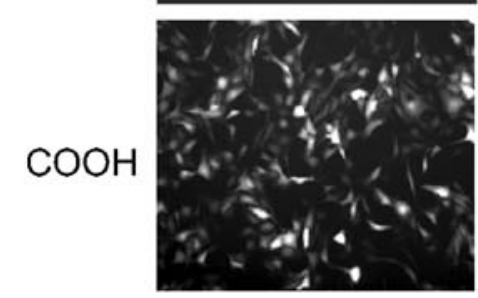

$\mathrm{COOH}$

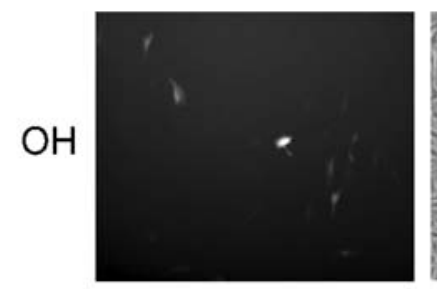

(b)

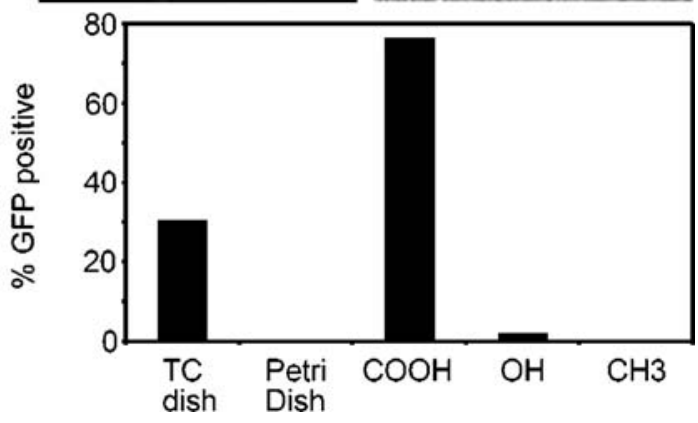

Fig. 2 Surface chemistry governs adenoviral immobilization. (a) Fluorescence and phase contrast images of surface immobilized adenoviral transduction of BPAEC on tissue culture dish, Petri dish, $\mathrm{COOH}$ terminated alkanethiol, or OH-terminated alkanethiol surfaces. Bar $100 \mu \mathrm{m}$. (b) Graph of surface immobilized adenovirus transduction efficiency in BPAEC on different surfaces

nant adenovirus. We observed that adenovirus was effectively immobilized on the $\mathrm{COOH}$ SAM, where approximately $80 \%$ of cells expressed the GFP marker (Fig. 2(a) and (b)). In contrast, cells poorly incorporated and expressed the GFP adenovirus on the $\mathrm{OH}$ SAM (Fig. 2(a) and (b)), and expression was undetectable on the $\mathrm{CH}_{3}$ SAM (Fig. 2(b) and data not shown). It has been previously suggested that the $\mathrm{CH}_{3}$ functionality interacts with proteins primarily through van der Waals interactions, while the $\mathrm{COOH}$ functionality interacts additionally through stronger polar (dipole-dipole, hydrogen bond, and ionic) interactions (Israelachvili 1991; Tan et al. 2002). One possibility is that such polar interactions are also at work in the context of adenoviral immobilization, and the $\mathrm{OH}$ functionality may have been less effective in adenoviral immobilization than the $\mathrm{COOH}$ functionality. The nonpolar $\mathrm{CH}_{3}$-terminated alkanethiol was either completely ineffective at immobilizing virus or denatured the virus upon immobilization. Regardless of the mechanism, these studies suggest that the surface chemistry of a substrate is a critical determinant of the immobilization of active adenovirus and further suggests that the immobilization of recombinant adenovirus onto a SAM surface can be tuned by adjusting the hydrophobicity of the terminal group and by altering the ratio of alkanethiols on the surface.

3.3 Patterning recombinant adenovirus results in patterned gene expression

Based on our findings that different surface chemistries have different capacities to immobilize adenovirus, we sought to exploit these differential immobilization capabilities to pattern gene expression within a monolayer of cells. To do this we stamped $150 \mu \mathrm{m}$ square islands of $\mathrm{COOH}-$ terminated alkanethiol onto a gold-coated coverslip, after which we immersed the coverslip in $\mathrm{CH}_{3}$-terminated alkanethiol so that all non-stamped regions would become $\mathrm{CH}_{3}$-functionalized (Fig. 3(a)). The coverslip was immersed in a $1 \times 10^{11}$ particles $/ \mathrm{mL}$ solution of adenovirus and following extensive washing, the coverslip was coated with fibronectin. A monolayer of cells was plated onto the coverslip and GFP expression was monitored at $24 \mathrm{~h}$ post seeding. In these experiments, we observed that cells did not adhere well to the $\mathrm{CH}_{3}$-functionalized regions of the coverslip, resulting in a non-uniform distribution of cells (Fig. 3(b) and (c)). These data indirectly suggest that FN is not adequately immobilized on the surface in the face of competing pre-adsorption by adenoviral protein (Chen et al. 1998; Tan et al. 2002). Thus, this approach generated an insufficiently uniform monolayer of cells within which patterned regions of gene expression could be controlled. To circumvent these shortcomings, we employed an alternative strategy based on masking to restrict adenovirus deposition. Here, a thin PDMS membrane containing holes was used to pattern adenovirus to discrete regions of a culture dish. These membranes were generated by spin coating a thin layer of PDMS over a silicon master with raised features (Ostuni et al. 2000). After curing and peeling, the membrane was sealed against a tissue culture dish and adenovirus was immobilized on the dish through the holes in the membrane. Following incubation with recombinant adenovirus, the dish was coated with fibronectin and cells were seeded in a monolayer. Using this 

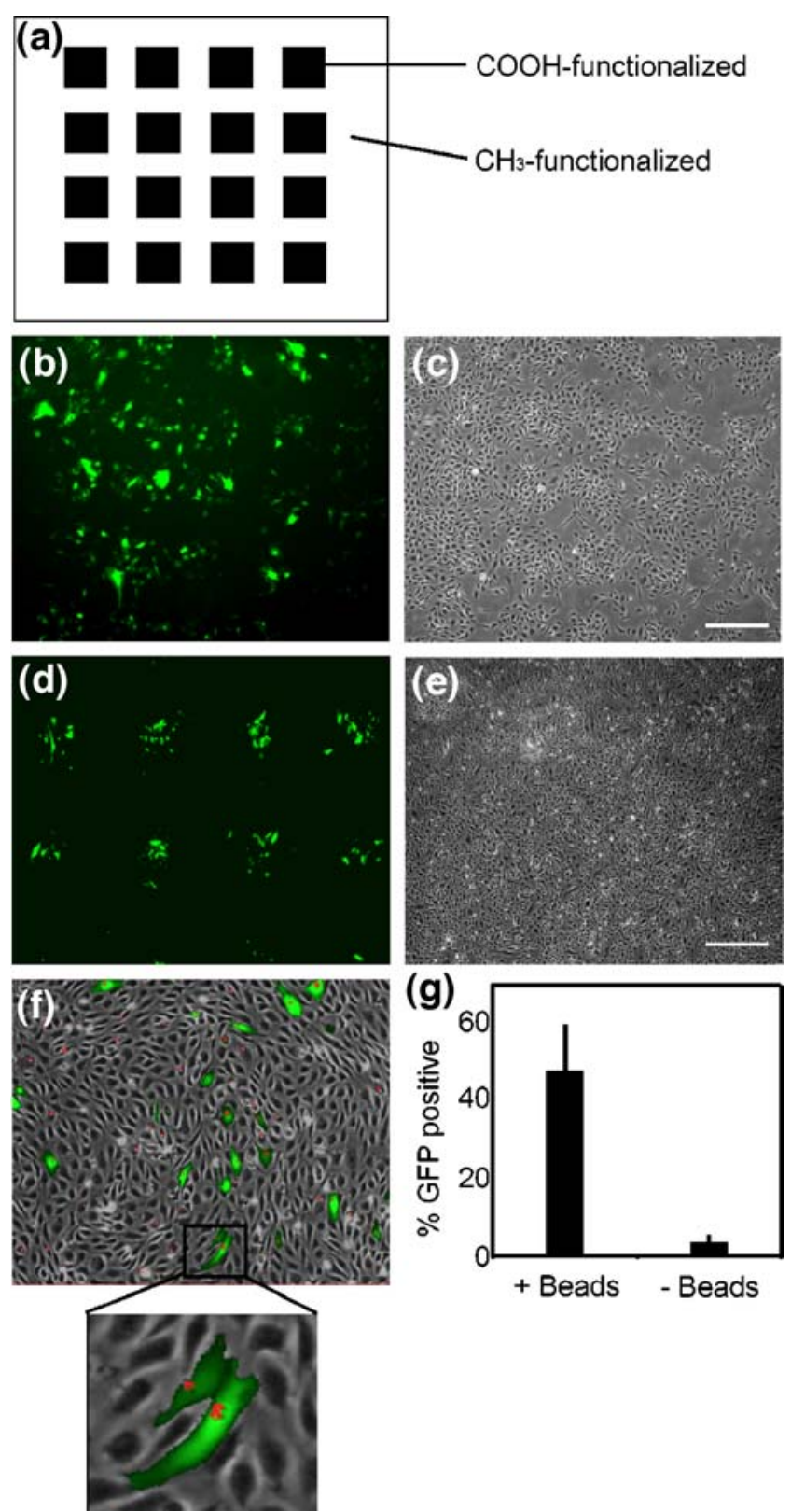

Fig. 3 Patterning of recombinant GFP adenovirus. $\mathrm{COOH}$-terminated alkanethiol islands were stamped onto gold-coated coverslips that were subsequently coated with a $\mathrm{CH}_{3}$-terminated alkanethiol to cover non-stamped regions (a). BPAECs were grown in a monolayer over the adenovirus adsorbed surface and GFP expression was monitored at $24 \mathrm{~h}$; Fluorescent image (b) and phase contrast image (c); Bar $200 \mu \mathrm{m}$. PDMS membranes containing $150 \mu \mathrm{m}^{2}$ holes were used to pattern adenoviral immobilization; Fluorescent image (d) and phase contrast image (e); Bar $200 \mu \mathrm{m}$. Combined fluorescent and phase contrast image of bead-immobilized adenovirus delivery (f). Image was pseudocolored to show GFP expressing cells (green) and beads (red) in $\mathbf{f}$. Graph showing specificity of viral transduction to cells in contact with bead-immobilized GFP adenovirus (g)

method, we were able to generate discrete regions of cells expressing GFP within a monolayer of cells (Fig. 3(d) and (e)). This approach highlights the ability to trigger gene expression within an otherwise homogenous population of cells.

\subsection{Beads as a viral delivery system}

While patterning surfaces provides one strategy to control gene expression in specific cells, we explored whether immobilizing virus on materials that subsequently can be delivered to cells also could be exploited for control of gene expression. The ability of adenovirus to immobilize on carboxylated surfaces was used to generate viral coated beads as a virus delivery system. Here, $6 \mu \mathrm{m}$ carboxylterminated polystyrene beads were incubated with the GFPexpressing recombinant adenovirus, washed, and delivered to a monolayer of endothelial cells (Fig. 3(f)). We observed high fidelity of bead-induced gene expression, where cells with beads attached showed approximately 50\% transduction efficiency, while there were very few cells expressing GFP that did not have attached beads (Fig. 3(g)). Such a system has important implications for selective gene delivery in culture and in vivo. Beads or other carrier-materials could be generated that contain immobilized adenovirus. Targeting of these materials in vivo would likely result in the limited expression of the gene product to discrete regions as we have seen in an in vitro setting.

\section{Discussion}

Over the last decade, engineering surface chemistries has proven to be a powerful tool to pattern cellular microenvironments. Such technologies have enabled the placement of cells in discrete areas with fine spatial control. Here we have investigated whether we could pattern gene expression rather than cells by manipulating surface chemistry to selectively immobilize recombinant adenovirus. Using alkanethiols on gold, we have developed a strategy to immobilize recombinant adenovirus that allows us to direct gene expression. Adenoviral transduction is effective in a broad range of cell types, even primary cells commonly used in drug discovery and tissue engineering. We have also expanded this method into a bead-based viral delivery system, which may be useful for the targeted delivery of recombinant adenovirus to specific in vivo targets. This technology has applications for studying cell function in vitro and in vivo, and holds promise for tissue engineering applications.

The field of tissue engineering is rapidly developing and holds much promise for the in vitro generation of structurally and functionally faithful tissues. In order to obtain functional engineered tissues the proper spatial patterning of cells is critical, and this ability to control cell position has dramatically improved in recent years due to the application of microfabrication-based technologies (reviewed in (Liu and Chen 2005)). Lessons from embryonic development would dictate that spatial control of gene 
expression is also critical for tissue development and function, though little has been done to spatially manipulate gene expression. Traditional ways to manipulate gene expression have not been used to control cell function spatially. For instance, one can create gene overexpression or knockdown by transfecting mammalian expression plasmids or siRNAs, respectively, but such gene expression manipulations are spatially random on a cell-by-cell basis. There have been similar difficulties using viral transduction, where depending on the amount used, viral transduction is either random in a subset of the cellular population, or uniform if higher amounts of virus are used. In the work presented here, we have demonstrated that recombinant adenovirus can be selectively immobilized depending on the surface chemistry of the underlying substrate. This effect can be combined with physical barrier based strategies to create more complex spatial patterns that have tunable immobilization capabilities. If combined with siRNA-based methods, this offers the ability to turn on or turn off a gene in a spatially controlled fashion. Together these technologies have the potential to provide insights into the organization of multicellular structures and ultimately may help guide tissue engineering strategies.

A bead-based viral delivery system also has potential gene therapy applications where adenovirus can be delivered to discrete regions of an in vivo environment. While adenoviruses have been demonstrated to have some clinical success (reviewed in (Douglas 2007)), one major drawback to their use is the potential to induce an inflammatory response. At best, this limits the duration of exposure (approx. 2-4 weeks) and at worst can lead to a lifethreatening immune response and even death (Crystal et al. 1994; O'Brien and Simari 2000). Adenoviral immobilization strategies like the one presented here may provide a way to target adenovirus to particular regions, while not affecting other nearby cells.

Acknowledgements This work was supported by a grant from the National Institutes of Health, National Institute of Biomedical Imaging and Bioengineering (C. Chen) and a Ruth L. Kirschstein NRSA fellowship (D. Pirone).

\section{References}

A. Abzhanov, C.J. Tabin, Dev Biol. 273, 134-48 (2004)

D.R. Albrecht, G.H. Underhill, T.B. Wassermann, R.L. Sah, S.N. Bhatia, Nat Methods, 3, 369-75 (2006)

S.N. Bailey, S.M. Ali, A.E. Carpenter, C.O. Higgins, D.M. Sabatini, Nat Methods, 3, 117-22 (2006)

S.N. Bhatia, U.J. Balis, M.L. Yarmush, M. Toner, Biotechnol Prog, 14, 378-87 (1998)

C.S. Chen, M. Mrksich, S. Huang, G.M. Whitesides, D.E. Ingber, Science, 276, 1425-8 (1997)

C.S. Chen, E. Ostuni, G.M. Whitesides, D.E. Ingber, Meth Mol Biol, 139, 209-219 (1998)

R.G. Crystal, N.G. McElvaney, M.A. Rosenfeld, C.S. Chu, A. Mastrangeli, J.G. Hay, S.L. Brody, H.A. Jaffe, N.T. Eissa, C. Danel, Nat Genet, 8, 42-51 (1994)

J.T. Douglas, Mol Biotechnol, 36, 71-80 (2007)

D. Hu, R.S. Marcucio, J.A. Helms, Development, 130, 1749-58 (2003)

M.J. Imperiale, S. Kochanek, Curr Top Microbiol Immunol, 273, 335 57 (2004)

J.N. Israelachvili, Intermolecular and Surface Forces, 2nd edn. (Academic, USA, 1991)

Y. Kawakami, J. Rodriguez-Leon, J.C. Belmonte, Curr Opin Cell Biol, 18, 723-9 (2006)

W.F. Liu, C.S. Chen, Materials Today, 12, 28-35 (2005)

G.P. Lopez, M.W. Albers, S.L. Schreiber, R. Carroll, E. Peralta, G.M. Whitesides, J Am Chem Soc, 115, 5877-5878 (1993)

Y. Maeda, V. Dave, J.A. Whitsett, Physiol Rev, 87, 219-44 (2007)

R. McBeath, D.M. Pirone, C.M. Nelson, K. Bhadriraju, C.S. Chen, Dev Cell, 6, 483-95 (2004)

M. Mrksich, L.E. Dike, J. Tien, D.E. Ingber, G.M. Whitesides, Exp Cell Res, 235, 305-13 (1997)

C.M. Nelson, R.P. Jean, J.L. Tan, W.F. Liu, N.J. Sniadecki, A.A. Spector, C.S. Chen, Proc Natl Acad Sci U S A, 102, 11594-9 (2005)

T. O'Brien, R.D. Simari, Mayo Clin Proc, 75, 831-4 (2000)

E.N. Olson, Science, 313, 1922-7 (2006)

E. Ostuni, R. Kane, C.S. Chen, D.E. Ingber, G.M. Whitesides, Langmuir, 16, 7811-7819 (2000)

D.M. Pirone, W.F. Liu, S.A. Ruiz, L. Gao, S. Raghavan, C.A. Lemmon, L.H. Romer, C.S. Chen, J Cell Biol, 174, 277-88 (2006)

K.L. Prime, G.M. Whitesides, Science, 252, 1164-7 (1991)

A.E. Serls, S. Doherty, P. Parvatiyar, J.M. Wells, G.H. Deutsch, Development, 132, 35-47 (2005)

R. Singhvi, A. Kumar, G.P. Lopez, G.N. Stephanopoulos, D.I. Wang, G.M. Whitesides, D.E. Ingber, Science, 264, 696-8 (1994)

J.L. Tan, J. Tien, C.S. Chen, Langmuir, 18, 519-523 (2002)

J. Ziauddin, D.M. Sabatini, Nature, 411, 107-10 (2001) 